\title{
Beitrag zur Kenntnis arktischer Sedimente
}

\author{
Von Paul W. Thomson, Bonn
}

1. Die Dryastone. In den noch unter mehr oder weniger arktischen Bedingungen entstandenen lakustrinen Bildungen sind die sog. Dryastone im Ostseebecken sehr verbreitet. Typische organogene Seeablagerungen: Gyttjen und Dybildungen, findet man ja erst von der Klimazone der geschlossenen subarktischen Wälder an. Auffallend ist in diesen Tonen stellenweise ein Reichtum an Blättern von: Dryas octopetala, Ericaceen wie Arctostaphylus alpina, Loiseleuria procumbens, Weiden wie Salix polaris, S.herbacea, S.reticulata usw., Betula nana und vielen anderen. Die meisten dieser Pflanzen wachsen nicht unmittelbar an den Ufern der Seebecken oder Bäche, die von Carex und Eriophorum-Beständen eingenommen werden. Es handelt sich um die Halbsträucher, die weitgehend die trockeneren Tundraflächen bedecken, die feuchteren Gebiete aber mehr oder weniger meiden. Zum Teil sind es, wie Salix berbacea, Schneetälchenpflanzen. Falls in den Bächen ein größerer Kalkgehalt im Wasser vorhanden ist, können auch gelegentlich Dryassträucher unmittelbar am Wasser wachsen. In der Regel ist aber Dryas octopetala eine Pflanze der kalkreichen, mehr oder weniger alkalischen Felsböden, während Loiseleuria procumbens die sauren Gesteinsböden bevorzugt. In Sommer 1933 und 1937 habe ich weite Wanderungen durch Schwedisch-, Finnisch- und Norwegisch-Lappland machen können und auch das damals finnische Petsamogebiet und die Fischerhalbinsel besuchen können. Gerade in Petsamo, einem größtenteils mit Moränenschutt bedeckten Tundragebiet, in dem basische Gesteine verbreitet sind, habe ich Ende Juli, Anfang August in nach N. exponierten Mulden reichlich Schnee gefunden. Dieser Schnee war volikommen mit Blättern der Felsbodentundragewächsen durchsetzt: Arctostaphylus alpina, Salix myrsinites, S.glauca, S.hastata, S.lanata, Dryas usw. Die über die Tundraflächen brausenden Stürme dürften hier die Blätter im Frühwinter mit dem Schnee zusammengewirbelt haben. Die von Tälchen ausgehenden Bäche führen nun diese Blätter in die in den Senken vorhandenen größeren und kleineren Seebecken. So ist es zu erklären, daß sich in diesen Seebecken, die von einer Carex-EriophorumVegetation umgeben sind, tonige Sedimente bilden, die in Massen Blätter der umgebenden Felsvegetation enthalten. So dürften auch die spätglazialen Dryastone entstanden sein, die sich nach dem Eisfreiwerden des Landes in den Senken des mehr oder weniger hügeligen Grundmoränengeländes im Ostseebecken gebildet haben (THOMson 1933).

2. Während der postglazialen Wärmezeit war der subarktische Waldgürtel mit den für ihn bezeichnenden Aapamooren und ähnlichen Bildungen weit in das heutige Tundragebiet vorgestoßen. Während der kühleren "Nachwärmezeit" zog sich die Baumgrenze nach $S$ zurück, und in den Mooren kam es zu den bekannten Auffrierungsund Abbauerscheinungen, die als Torfhügel oder Palsen bekannt sind. Auf die große Literatur darüber möchte ich nicht näher eingehen. Die vorzüglichen Arbeiten von V. AuER und die zahlreichen der russischen Literatur seien nur kurz erwähnt.

Im Sommer 1937 habe ich im Enontekiogebiet in der Nähe des Kilpisjärvi, wo Finnland, Schweden und Norwegen zusammenstoßen, wundervoll entwickelte Torfhügel von etwa $7 \mathrm{~m}$ Höhe beobachten können. Es herrschte damals eine Trockenheit von mehreren Wochen. Die vegetationsfreien Oberflächen der Torfhügel waren weitgehend trocken, so daß der Torf im Winde verweht wurde und richtige Torfmullwehen zustande kamen. In den Senken kann es dann zu Sedimenten kommen, in denen der vom Winde verwehte Torfmull mit Ton vermengt abgelagert wird. Da das Material in den Torfhügeln, wie es V. AUER so schön nachgewiesen hat, langsam von unten nach oben wandert, so werden während des Abbaues verschiedene Horizonte des Torflagers durch 
Winderosion abgetragen. Die heutigen Palsen finden sich ja nur in der schmalen Zone, innerhalb deren die Waldgrenze während der Postglazialzeit geschwankt hat.

Von ganz anderem Ausmaße muß dieses Palsenphänomen zu Beginn einer Eiszeit gewesen sein. Hier gerieten riesige Moorkomplexe mit mächtigen Hoch- und Niedermoortorflagern in den Bereich des Abbaues. In "Eiszeitalter und Gegenwart" (Тномson 1951) habe ich derartige Sedimente im Hangenden des anstehenden Eeminterglazials beschrieben und gezeigt, daß in ihnen zuerst die Hochmoorelemente der ursprünglichen Oberflächen der umgebenden Moore zur Ablagerung kamen, später auch die tieferen wärmezeitlichen Schichten. Deren Pollenführung kann eine Klimaschwankung vortäuschen, wenn man die Entstehung des Sediments nicht berücksichtigt. In Estland (THомson 1942) habe ich sogar über dem anstehenden Interglazial eine mächtige allochthone Schichtenserie beobachtet (vgl. ORvikU 1939), die aus Hoch- und Niedermoorelementen bestand und Gyttjaklumpen enthielt. Die letzteren stammten aus der Basis der abgebauten Torflager und zeigten eine ältere, z. T. wärmezeitliche Pollenführung. Solche Sedimente dürften im Hangenden von Interglazialen weit verbreitet sein, und so weit sie nicht erkannt werden, eine zweite Wärmezeit vortäuschen.

3. Wie schon erwähnt, herrschen unter arktischen Bedingungen klastische Ablage. rungen vor, wie die Dryastone usw. Während der Allerödzeit hat nun eine beträchtliche Schwankung der Waldgrenze nach $\mathrm{N}$ und W stattgefunden. In diesen Gebieten ist die bekannte Schichtenfolge zu verzeichnen:

\section{Jüngere Dryastone \\ Allerödgyttja \\ Altere Dryastone.}

Im Ostbaltikum ist diese Schichtenfolge typisch für das Gebiet von Litauen resp. Süd-Lettland bis über Ostpreußen nach S zu (Gross 1937). Das zweite Vorkommen dieser klassischen Allerödschwankung nach dem locus classicus in Dänemark habe ich mit K. Brundza in Litauen feststellen können (G. F. F. 1931. 1935). In Estland habe ich mich im Laufe von 20 Jahren mit quartärgeologischen Studien befaßt und hunderte von Moor- und Seekomplexen untersucht. Hier fehlt der Waldvorstoß während der Allerödzeit. Es dürfte hier auch während der Allerödwärmezeit die Tundra weitgehend geherrscht haben, wenn auch an geschützten Stellen größere oder kleinere Waldinseln vorhanden gewesen sein könnten. Ich habe in Estland in spätglazialen Tonfolgen wohl gelegentlich dunklere Streifen gefunden, die reich an organischer Substanz waren, eine Waldbedeckung ließ sich aber nicht nachweisen. Trotzdem führen die spätglazialen Tone auch am Südufer des Finnischen Meerbusens reichlich Fichtenpollen und sogar Fichtenholz, gleichzeitig auch den Pollen wärmezeitlicher Bäume und sogar von Carpinus, der im Eeminterglazial in Estland mit Frequenzen von 31-38\% vorkam (Carpinus-Früchte zusammen mit Früchten von Brasenia purpurea, Najas marina usw., Thомson 1942). Es handelt sich hier, wie z. B. in Kunda im N.O. Estlands, eindeutig um sekundären Pollen, der aus dem Eeminterglazial stammt. Am Boden des Finnischen Meerbusens sind größere Interglazialvorkommen vorhanden. Damit stehen in Verbindung auch die Mammutfunde (Incisoren und Molare), die am Südufer des Finnischen Meerbusens in der Grundmoräne gemacht worden sind und aus der Endphase des Eeminterglazials stammen dürften.

Von E. Dreimanis und auch F. Zeuner-London sind die von mir in Kunda beschriebenen spätglazialen Tone als typisches Alleröd gedeutet worden. Es handelt sich zweifellos um Ablagerungen, die auch die Allerödzeit zeitlich umfassen, die aber hier auch während der Allerödwärmezeit einen Tundracharakter hatten. Der Fichtenpollen und der wärmezeitlicher Bäume ist sekundär, wie z. B. der miozäne Pollen, der vielfach in Schleswig-Holstein und Niedersachsen in den älteren Dryastonen vorherrscht (IVersen 1936, Thomson 1933). 
Im Warthegebiet habe ich keine typische Allerödfolge feststellen können. In Kujawien dürfte schon während der Zeit der älteren Dryastone bereits eine Waldbedeckung geherrscht haben. Etwas höher wird die Kiefer gegenüber der Birke vorherrschend. Dann folgt in vielen Profilen wieder eine Zunahme der Birkenkomponente. Dieser Abschnitt dürfte mit großer Wahrscheinlichkeit nach der Jüngeren Dryaszeit entsprechen (unveröffentlichte und durch den Krieg vernichtete Arbeiten von Frl. Helma SJUTs).

Das Gebiet, in dem die klassische Allerödabfolge zu beobachten ist, dürfte östlich der Ostsee vom südlichen Lettland über Litauen und Ostpreußen bis weit nach Polen gereicht haben. Im Kujawischen Gebiet dürften die ganze Zeit hindurch Birken-Kiefernwälder vorhanden gewesen sein, und nur eine vorübergehende Zunahme der Kiefernfrequenz dürfte hier die Allerödzeit anzeigen.

In der Frühwärmezeit bis in die Wärmezeit hinein dürften im kujawischen Schwarzerdegebiet steppenartige Pflanzenvereine auf den Grundmoränen-Plateaus geherrscht haben und nur in den Talsandgebieten Kiefernwälder mit Elementen des Eichenmischwaldes. Das Querceto-Carpinetum dürfte hier im wesentlichen in der Spät- und Nachwärmezeit auf den reichen Grundmoränenböden zur Herrschaft gelangt sein.

In maritimen Gebieten reicht die Waldgrenze weniger weit nach Norden als in den kontinentalen derselben Breite. Das Gebiet mit der „klassischen Allerödfolge“ reicht daher hier weiter nach Süden. Das Böllingstadium dürfte, soweit mir bekannt, vielleicht an dieses Gebiet gebunden sein und könnte gut eine $Z_{w}$ eiteilung des im kontinentaleren Osten einheitlichen "Alleröds“ darstellen.

\section{Literaturverzeich is}

Dreimanis, A.: A Draft of Pleistocene Stratigraphy in Latvia and S. Esthonia. - G. F. F. Stockholm 69, 1947.

FAEGRI, K.: Quartärgeologische Untersuchungen im westlichen Norwegen. I. Über zwei präboreale Klimaschwankungen im südwestlichen Teil. - Bergens Museum Arbok, Naturv. Raekke Nr. 8. 1935.

Gross, H.: Nachweis der Allerödschwankung im Süd- und Ostbaltischen Gebiet. - Beih. Botan. Zentrbl. 5\%, Berlin 1937.

Iversen, J.: Sekundäres Pollen als Fehlerquelle. - Danmarks Geol. Unders. IV. Raekke Bd. 2, Nr. 15. 1936.

Jessen, K.: Moseundersogelser i det nordostlige Sjaelland. - Danmarks Geol. Unders. II. 34. Kopenhagen 1920.

NiLsson, T.: Die pollenanalytische Zonengliederung der spät- und postglazialen Bildungen Schonens. - G. F. F. 57. Stockholm 1935.

Orviku, K.: Das Interglazial von Ringen-Röngu. - Eesti Loodus, Dorpat 1939

Thomson, P. W.: Die regionale Entwicklungsgeschichte der Wälder Estlands. - Acta et Comm. Univ. Tartuensis (Dorpatensis) A. XVII. Tartu - Dorpat 1929. - - Zur Genesis der Dryastone. Beobachtungen im Petsamogebiet in Lappland. - Beiträge zur Kunde Estlands 18. Reval 1933. - - Beitrag zur Stratigraphie der Moore und zur Waldgeschichte Südwest-Litauens. - G. F. F. 53. Stockholm 1931. - - Úber Mammutfunde in NordEstland. - Eesti Loodus. Dorpat 1934. - - Vorläufige Mitteilung über die spätglaziale Waldgeschichte Estlands. - G. F. F. 5\%, 1933. - - Die Klima- und Waldentwicklung des von K. Orviku entdeckten Interglazials von Ringen bei Dorpat/Estland. Z. deutsch. geol. Ges. 93, 1941. - - Das Interglazial von Wallensen im Hils. - Eisz. u. Gegenwart 1, 1951.

Manuskr. eingeg. 27. 3. 1955

Anschrift d. Verf.: Prof. Dr. P. W. Thomson, Bonn, Belderberg 4. 